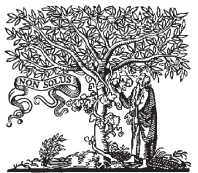

EI SFVIER

\title{
Substance use in young adults: Associations with personality and gender
}

\author{
Todd B. Kashdan, Charlene J. Vetter, R. Lorraine Collins* \\ Department of Psychology, Research Institute on Addictions, University at Buffalo, State University of New York, \\ 1021 Main Street, Buffalo, NY 14203, USA
}

\begin{abstract}
We examined relationships among personality (i.e., negative affectivity and conscientiousness), and use of licit and illicit substances in a sample of 421 college-aged social drinkers (52.7\% women, $47.3 \%$ men). Results indicated significant relationships between personality and substance use as well as gender differences. Negative affectivity was related to greater illicit substance use, but not alcohol use or smoking. Conscientiousness was related to less alcohol use and smoking, which fully mediated relationships between conscientiousness and with less use of marijuana and other illicit substances. For women, conscientiousness was associated with less alcohol and smoking, compared to men. For men, alcohol use and smoking were more likely to lead to marijuana use, compared to women. Our findings support differential pathways from personality to substance use, and gender appears to be an important moderating factor.
\end{abstract}

(C) 2004 Elsevier Ltd. All rights reserved.

Keywords: Personality; Gender; Alcohol; Smoking; Marijuana; Illicit substances

\section{Introduction}

Drinking alcohol and smoking tobacco may increase the risk of progression to experimental, occasional, and regular use of illicit substances, such as marijuana and cocaine (Chen \& Kandel, 1995; Duncan, Duncan, \& Hops, 1998; Wagner \& Anthony, 2002). Nonetheless, many adolescents and young adults who experiment with alcohol and smoking do not progress to using illicit substances. We hypothesized that specific dispositional sensitivities would further elucidate pathways to alcohol and smoking, and illicit substance use.

* Corresponding author. Tel.: +1-716-887-2523; fax: +1-716-887-2252.

E-mail addresses: kashdan@buffalo.edu (T.B. Kashdan), collins@ria.buffalo.edu (R.L. Collins). 
Negative affect (NA), alcohol use, and smoking are positively associated (e.g., Dierker, Avenevoli, Stolar, \& Merikangas, 2002; Wills, Sandy, \& Yaeger, 2002). Those high in NA consume more substances in their efforts to reduce negative states or increase positive states (Cooper, Frone, Russell, \& Mudar, 1995). For example, adolescents who smoke regularly report higher levels of depression and anxiety than occasional smokers (Patton et al., 1996), and NA is related to increases in smoking over time (Wills et al., 2002).

In contrast, individuals who are conscientious, self-disciplined, and low in impulsivity report less alcohol use and smoking, which may decrease their likelihood of using illicit substances. Highly conscientious individuals have greater feelings of personal control and are more apt to pursue and attain meaningful life goals. The greater self-regulatory strength of highly conscientious individuals is expected to offer protection against risky health behaviors (Friedman et al., 1995). Specifically, such individuals have a greater likelihood of exerting self-control when exposed to substances and therefore are less likely to use them or better able to regulate their substance use (Adalbjarnardottir \& Rafnsson, 2001; Finn, Sharkansky, Brandt, \& Turcotte, 2000; Wills, Sandy, \& Yaeger, 2000). We expected conscientiousness to be associated with less alcohol use, smoking, and illicit substances. In addition, relationships between conscientiousness and illicit substances were expected to be mediated by alcohol use and smoking.

In the present study, we explored the associations among the dispositional sensitivities of trait NA and conscientiousness with substance use. Based on gender differences in substance use and personality, we also explored gender differences in these pathways. For example, women consume less alcohol and are less likely to use illicit substances than do men (Filmore et al., 1997; Johnston, O'Malley, \& Bachman, 2002). Women show a greater propensity to experience NA (e.g., Nolen-Hoeksema \& Girgus, 1994), and depressed mood and alcohol consumption appear to be more strongly related in female than male adolescents (Locke \& Newcomb, 2001). Thus, NA may place some women at increased risk for substance use. On the other hand, women report greater conscientiousness than men (Costa, Terracciano, \& McCrae, 2001) and conscientiousness offers some protection against substance use (Wills et al., 2000). Thus, conscientiousness may be more protective against substance use for women than men.

Typically, marijuana is aggregated with other illicit substances, such as cocaine and amphetamines. However, the social and legal status of marijuana is not the same as other illicit substances. Marijuana use is highly prevalent, social attitudes towards its use are more permissive, and it has been legalized for medical use in some western states of the United States. For these reasons, we explored whether marijuana should be treated as independent from the other illicit substances in this study, namely, cocaine, tranquilizers, amphetamines, and opiates.

We used structural equation modeling techniques to examine our hypotheses. Both NA and conscientiousness have shown relationships with alcohol, smoking, and illicit substances, and so we examined their unique predictive utility by controlling for shared variance. NA was expected to have direct positive relationships with licit (i.e., alcohol and smoking) and illicit substances. In contrast, conscientiousness was expected to have a negative relationship with alcohol use and smoking, and they in turn were expected to fully 
mediate the relationship between conscientiousness and illicit substance use. Finally, we expected NA and conscientiousness to have stronger relationships with substance use in women compared to men.

\section{Method}

\subsection{Participants}

Young adult social drinkers were recruited using newspaper advertisements and flyers. Inclusion criteria were age (18-20 years), minimum 4-11 drinks/week, and no prior alcoholor substance-related disorders, treatment, or legal consequences. The final sample $(N=421)$ included 222 women (52.7\%) and 199 men (47.3\%), with a mean age of 19.03 (S.D.=.79) years. The majority was single $(99.8 \% ; n=420)$, from a European American background $(87.6 \% ; n=369)$, and enrolled in postsecondary education $(92.6 \% ; n=390)$. Small groups completed a randomly ordered battery of questionnaires, from which we selected the following relevant measures.

\subsection{Measures}

\subsubsection{Negative affectivity}

Participants used a five-point Likert scale to rate the 12 items of the neuroticism subscale of the NEO-Five Factor Inventory (NEO-FFI; Costa \& McCrae, 1991; $\alpha=.83$ ), which assesses general tendencies to experience NA. They used a four-point Likert scale to rate the 21 items of the Beck Depression Inventory-II (BDI-II; Beck, Steer, Ball, \& Ranieri, 1996; $\alpha=.88$ ), which assesses depressive symptoms during the past 2 weeks.

\subsubsection{Conscientiousness}

Participants used a five-point Likert scale to rate the 12 items of the conscientiousness subscale of the NEO-FFI (Costa $\&$ McCrae, 1991; $\alpha=.83$ ) to assess general tendencies to be disciplined, self-controlled, dependable, and orderly.

\subsubsection{Alcohol use}

Use of all substances was assessed using the General Information Questionnaire (GIQ). Participants reported their typical intake of standard drinks (e.g., 12-oz beer, 4-oz wine, and 1-oz shot liquor) for each day of the week (cf. Collins, Parks, \& Marlatt, 1985). Daily intake was summed to derive a measure of weekly drinking.

\subsubsection{Use of tobacco and other substances}

For tobacco, marijuana, cocaine, tranquilizers, amphetamines, and opiates, participants indicated typical use on a four-point Likert scale (never used, in lifetime; rarely use, once or twice but not in past month; occasionally use, used during the past month, but not regularly; regularly use, use at least once a week or daily). 


\subsection{Overview of data analysis}

Nine participants ( $2 \%$ of the sample) failed to complete all of the measures under study and were dropped from our original sample of 430 underage drinkers. We used the final sample of 421 drinkers to test the validity of our path model with AMOS 4.0 (Arbuckle \& Wothke, 1999), using maximum likelihood procedures. We also tested the consistency of the measurement model across men and women. Standardized and unstandardized regression coefficients were computed for paths between variables.

\section{Results}

\subsection{Descriptive statistics}

Means and standard deviations for the variables of interest are reported in Table 1. Except for tobacco smoking and marijuana use, we logarithmically transformed substance use scores because of nonnormal distributions (absolute values for skewness ranged from 1.80 to 2.58 and kurtosis ranged from 4.91 to 6.95). The data were normalized such that absolute values for skewness ranged from -0.04 to 2.04 and kurtosis ranged from -0.08 to 2.89 .

\subsection{Associations among personality and substance use variables}

Table 1 shows that neuroticism and depression were positively correlated, $r \mathrm{~s}=.63, P<.001$. Conscientiousness was negatively correlated with neuroticism and the BDI-II, $r \mathrm{~s}=-.34$ and $-.35, P_{\mathrm{s}}<.001$. For women, depressive symptoms had small, positive relationships with substance use, except for alcohol and marijuana. In contrast, neuroticism had only small positive relationships to smoking, marijuana, and cocaine use. Conscientiousness was negatively related to substance use. Alcohol use and smoking were moderately positively related, $r=.26, P<.01$. Only a few significant gender differences were present. Neuroticism was significantly greater for women compared to men, $t(428)=-3.11, P<.005$. Average alcoholic drinks per week was greater for men compared to women, $t(428)=6.16, P<.001$. These findings are consistent with previous research and suggest that multicollinearity is not an issue in our data.

\subsection{Testing models of personality and substance use}

Our initial model (see Fig. 1) consisted of three latent variables: negative affectivity (neuroticism and depression), licit substance use (drinks/week and GIQ typical smoking item), and illicit substance use (GIQ typical use of marijuana, cocaine, amphetamines, and opiates). Conscientiousness was an observed variable, and NA and conscientiousness were set to covary. We hypothesized that NA would be directly related to licit and illicit substances and that licit substances would fully mediate the relationship between conscientiousness and illicit substances.

Our initial model was an adequate fit to the data, $\chi^{2}(23)=82.90, P<.001, \chi^{2} /$ $d f=3.60, \mathrm{CFI}=.99, \mathrm{NFI}=.99, \mathrm{RMSEA}=.08(90 \% \mathrm{CI}=.06-.10)$. Contrary to expectations, 
T.B. Kashdan et al. / Addictive Behaviors 30 (2005) 259-269

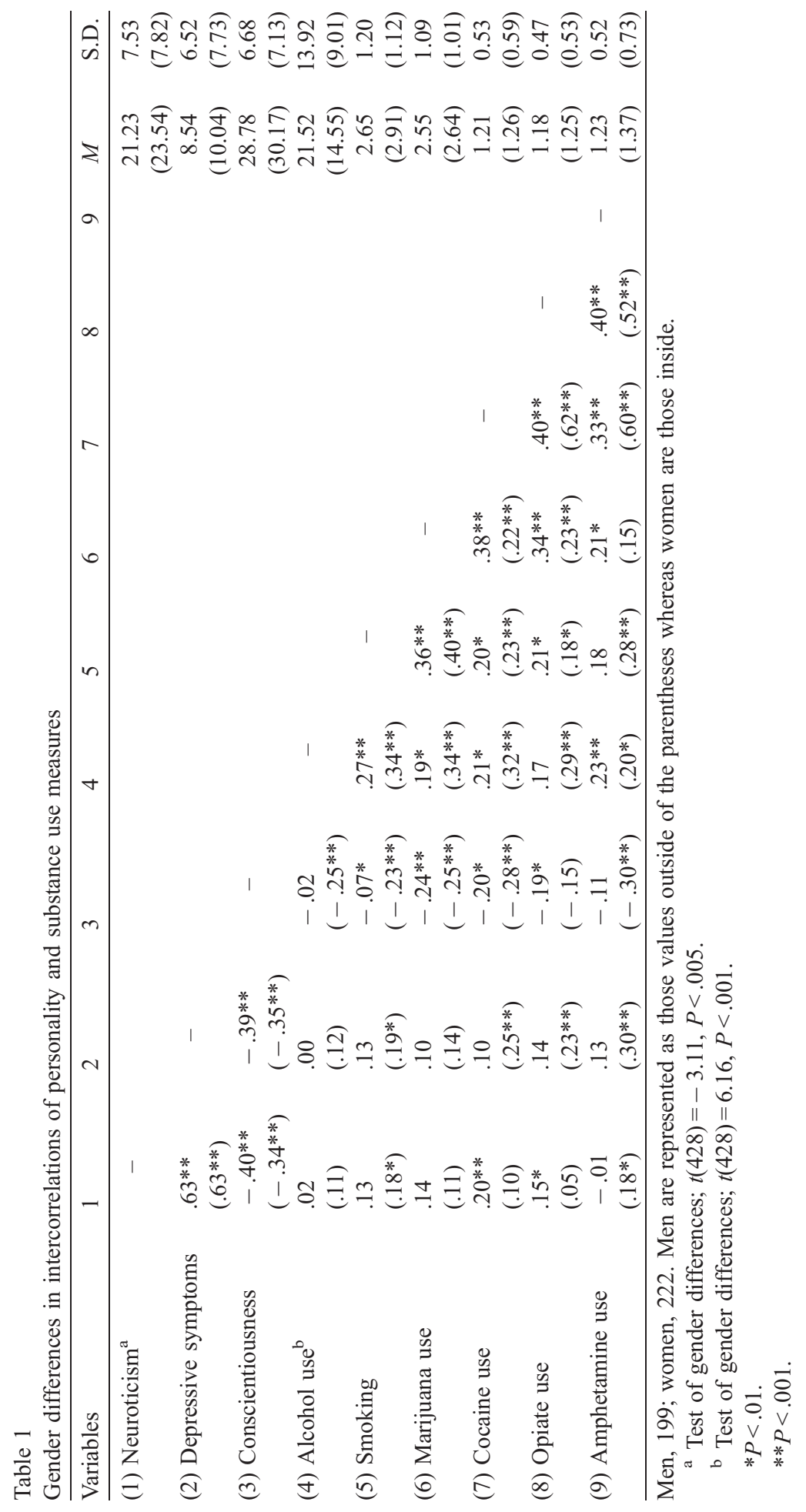



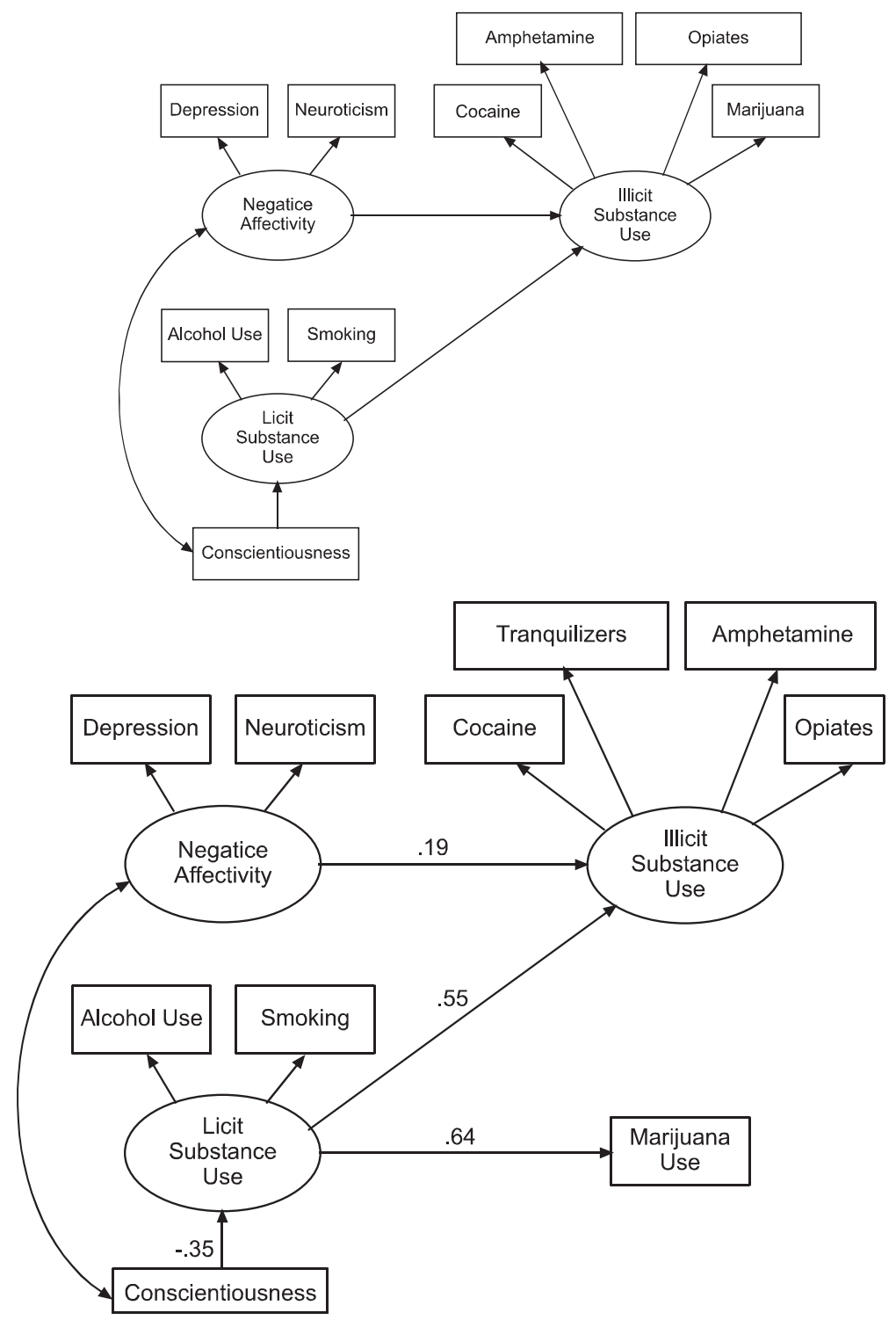

Fig. 1. Conceptual and final models of relationships among predictor variables and substance use outcomes. Note. $n=421$. Final model of the associations among personality traits, licit substance use, and illicit substance use; $€ 2$ $(24)=43.72, p<.01, € 2 / d f=1.82, C F I=1.00, N F I=.99, R M S E A=.04(90 \% \mathrm{CI}=.02-.06)$. The curved doubleheaded arrows indicate covariances and single-headed arrows indicate path effects. Latent variables were created from the following scales: (1) Negative Affectivity: Neuroticism (NEO-Five Factor Inventory; $B=.75$ ) and depressive symptoms (Beck Depression Inventory-II; $B=.84)$, (2) licit drugs: weekly intake of alcohol $(B=.44)$ and typical smoking $(B=.56)$, and (3) illicit drugs: typical use of cocaine $(B=.75)$, amphetamines $(B=.66)$, and opiates $(B=.70)$. The covariance between Negative Affectivity and Conscientiousness was -.43 . 
the path from NA to alcohol and smoking failed to reach significance, $B=.11(P>.15)$. Additionally, marijuana failed to adequately load on the latent construct of illicit substances, $B=.42$ (all other loadings $>.65$ ). The nonsignificant path from NA to alcohol and smoking was deleted to produce a more parsimonious, better fitting model. To further revise the model, marijuana was removed from the latent construct of illicit substances and retained as an independent observed variable predicted by alcohol and smoking.

Our revised model (Fig. 1) provided an excellent fit to the data; $\chi^{2}(24)=43.72, P<.01, \chi^{2} /$ $d f=1.82, \mathrm{CFI}=1.00, \mathrm{NFI}=.99$, and an RMSEA $=.04(90 \% \mathrm{CI}=.02-.06) . \mathrm{NA}$ and conscientiousness had a strong inverse relationship $(r=-.43, P<.001)$. Alcohol and smoking was a predictor of greater use of illicit substances $(B=.55, P<.001)$ and marijuana $(B=.64, P<.001)$. NA predicted greater illicit substances $(B=.19, P<.005)$, and conscientiousness predicted less alcohol use and smoking $(B=-.34, P<.001)$.

To examine whether alcohol use and smoking mediated the pathways from conscientiousness to illicit substances and marijuana, we examined alternative models with paths from conscientiousness to each of these outcomes. In these separate alternative models, adding paths from conscientiousness to illicit substances, $\chi^{2}(23)=43.27, P<.01$, and conscientiousness to marijuana use, $\chi^{2}(23)=42.91, P<.01$, led to near identical fits to our revised model. However, the path from conscientiousness to illicit substances $(P=.50)$ and conscientiousness to marijuana use $(P=.36)$ each failed to reach significance. Although each of these models had a similar fit to our revised model, they were less parsimonious. Supporting our initial hypotheses, we demonstrated that alcohol and smoking fully mediated relationships between conscientiousness and use of illicit substances and marijuana. Based on these results, we retained the final model in Fig. 1.

The fully revised model accounted for $12 \%, 37 \%$, and $41 \%$, respectively, of the residual variance in use of alcohol and smoking, illicit substances, and marijuana. The $12 \%$ variance explained in alcohol and smoking is the function of a single predictor, conscientiousness. Thus, the model appears to be considerably above the typical thresholds for satisfactory model fit and in addition, explains an acceptable level of variance in each of the endogenous variables.

\subsection{Test of gender differences}

To examine gender differences in our final model (Fig. 1), we analyzed all facets of the model using a multisample analysis in which subgroups of men and women were tested simultaneously. We tested gender differences by constraining certain paths to be equal. A hierarchy of hypotheses was conducted on (1) factor loading patterns, (2) factor variances/ covariances, and (3) structural pathways. Invariance tests of error variances were not included in this analysis because this set of hypotheses has been shown to be the least important, particularly in the absence of specific hypotheses (Bentler, 1995). Parameters found to be invariant across gender were cumulatively constrained before testing the structural paths (e.g., Byrne, 2001). This logically ordered set of gender difference tests is increasingly restrictive and conservative. Gender differences in factor loadings, variances, and covariances were all statistically controlled in subsequent S.E.M. analyses of gender 
Table 2

Goodness-of-fit tests of gender invariance for structural pathways

\begin{tabular}{|c|c|c|c|c|c|c|c|c|}
\hline \multirow[t]{2}{*}{ Model } & \multirow[t]{2}{*}{$\chi^{2}$} & \multirow[t]{2}{*}{$d f$} & \multirow[t]{2}{*}{$P$} & \multicolumn{3}{|c|}{$\begin{array}{l}\Delta \text { From baseline } \\
\text { model }\end{array}$} & \multicolumn{2}{|c|}{$\begin{array}{l}\text { Path coefficients from } \\
\text { separate sample analyses }\end{array}$} \\
\hline & & & & $\bar{\chi}^{2}$ & $d f$ & $P$ & Men & Women \\
\hline (1) Baseline model & 74.46 & 52 & $<.05$ & - & - & & & \\
\hline $\begin{array}{l}\text { (2) Negative affectivity to illicit } \\
\text { substance use }\end{array}$ & 75.05 & 53 & $<.05$ & 0.59 & 1 & $=.44$ & $(.13)$ & $(.13)$ \\
\hline $\begin{array}{l}\text { (3) Conscientiousness to licit } \\
\text { substance use }\end{array}$ & 77.53 & 53 & $<.05$ & 3.72 & 1 & $=.08$ & $.00(-.28)$ & $-.01 * *(-.42)$ \\
\hline (4) Licit to illicit use & 76.28 & 53 & $<.05$ & 1.82 & 1 & $=.18$ & $.63 * *(.72)$ & $.40 * * \quad(.47)$ \\
\hline (5) Licit substance use to marijuana use & 78.00 & 53 & $<.05$ & 3.54 & 1 & $=.06$ & $7.64 * *(.70)$ & $4.12 * * \quad(.60)$ \\
\hline
\end{tabular}

Men, 199; women, 222. In Models 2 through 5, the single pathway indicated was constrained to be equal between men and women.

${ }^{\text {a }}$ Unstandardized (standardized).

$* * P<.001$.

differences in structural pathways. ${ }^{1}$ This procedure provides evidence that gender differences in structural pathways are not a function of gender differences in other parts of the underlying theoretical structure (or instability in the model). Despite suggestions for using this iterative technique in testing multisample models (e.g., Bollen, 1989; Byrne, 2001), it is still not commonly used.

Upon testing measurement invariance across genders, the only source of discrepancy lay with the amphetamine factor loading of illicit substances. As a result of invariant factor loadings on latent variables, we were able to subsequently examine gender invariance in structural pathways (Byrne, 2001). Tests of gender invariance, reported in Table 2, held the following parameters equivalent between genders: neuroticism, opiate use, and tobacco use factor loadings, and the covariance between neuroticism and conscientiousness (parameters for depressive symptoms and alcohol and cocaine use were set to 1.0 which is why these variables were not examined for equivalence). As shown in Table 2, each of the structural pathways in our revised model was statistically invariant across gender. However, near significant decrements in overall model fit were found following the inclusion of equality restraints on paths from conscientiousness to licit substances (men; $B=-.28$; women; $B=-.42$ ) and licit substances to marijuana (men; $B=.70$; women; $B=.60$ ). Paths from conscientiousness to alcohol and smoking were stronger for women compared to men. Positive paths from alcohol use and smoking to marijuana were stronger for men than women.

\footnotetext{
1 The factor structure pattern for illicit substance use (Fig. 1) was similar for men and women. Upon testing measurement invariance across genders, sources of discrepancy lay with the smoking factor loading of licit substance use, tranquilizer and opiate factor loadings of illicit substance use, and covariance between negative affectivity and conscientiousness. As a result of invariant factor loadings on latent variables, we were able to subsequently examine gender invariance in structural pathways (e.g., Byrne, 2001). Details on analyses and results can be obtained by request.
} 
Finally, we examined the variance explained by the separate models for men and women. The separate models accounted for different levels of residual variance in alcohol use and smoking ( $8 \%$ for men; $18 \%$ for women), illicit substances ( $56 \%$ for men; $31 \%$ for women), and marijuana (48\% for men; $36 \%$ for women). Each of the models explains an acceptable level of variance in each of the endogenous variables, thereby providing further support for the importance of gender.

\section{Discussion}

Using data from underage social drinkers, we examined the role of dispositional sensitivities in substance use. Consistent with prior research, alcohol use and smoking in younger adults were significant predictors of use of illicit substances and marijuana (cf. Duncan et al., 1998). This occurred although marijuana did not load on the latent variable for illicit substances. As for specific hypothesized pathways, NA predicted greater use of illicit substances and conscientiousness predicted less alcohol use and smoking. High levels of conscientiousness buffered against alcohol and smoking directly and illicit substances and marijuana use indirectly, by its effect on alcohol and smoking. There also were some gender differences.

The pathway from conscientiousness to alcohol and smoking was stronger in women compared to men. The progression from alcohol and smoking to using marijuana and illicit substances was stronger in men compared to women. Hence, gender appears to be an important moderating variable in understanding the processes linking personality dispositions and substance use (Adalbjarnardottir \& Rafnsson, 2001).

Perhaps our most interesting findings were that NA was directly associated with greater illicit substances whereas the pathway from conscientiousness to illicit substances and marijuana use was mediated by alcohol use and smoking. These and other findings should be interpreted with caution because of the limitations in our study. The data is cross-sectional and based on self-reports, which limits our ability to make any causal interpretations. It seems logical to conclude that dispositional tendencies precede substance use. However, the differential pathways from NA and conscientiousness to substance use require validation in prospective studies in which the temporal precedence of personality factors can be established. Our tests of the unique variance attributable to NA and conscientiousness suggest that the robust relationship between NA and alcohol use and smoking is largely a function of shared variance with conscientiousness (or inversely, impulsivity). In contrast to the majority of studies that examine personality factors independently, there appears to be merit in examining the unique and shared variance of risk and protective factors in predicting substance use outcomes.

Our sample of young adults may be nonrepresentative because we set a minimum number (four) of drinks per week. This inclusion criterion probably led to an underestimation of the size of our effects. Better representation of the range of alcohol use, from abstainers through excessive drinkers, would likely lead to more valid tests of protective and risk factors. 
Technically, tobacco was the only substance that was legally available to our sample of young adults. However, we chose to treat alcohol as a licit substance because it is legally available to adults aged 21 years or older and is consumed by such large proportions of 18- to 20 -year-olds, that its use is considered normative among that age group (cf. Johnston et al., 2002). Furthermore, there are data to support treating an illicit drug, such as marijuana, as independent from alcohol use in college-aged samples (Katz, Fromme, \& D'Amico, 2000). Aggregating all of these substances into a single category of illicit substance use could have masked differential relationships, pathways, and trajectories.

Our preliminary findings have implications for developmental models of substance use. Future work can examine the behavioral tendencies, cognitive processes, and interpersonal networks of youth differing in conscientiousness and NA to further elucidate pathways to use of different substances. Reducing negative affective states, shown to be malleable in young adults, may offer protection against substance use and abuse. Cultivating conscientiousness may help to reduce alcohol use and smoking, as well as the progression to use of marijuana and other illicit substances. The interactive influence of gender and personality offers insight into understanding the substance use behaviors of young adults.

\section{Acknowledgements}

Portions of this article were presented at the 2002 convention of Association for Advancement of Behavior Therapy, Reno, NV. This study was supported by a NRSA predoctoral fellowship from the National Institutes of Health (F31 MH63565-01A1) to Todd B. Kashdan and a grant from the National Institute on Alcohol Abuse and Alcoholism/NIH (R01-AA07595) to R. Lorraine Collins. We would like to thank Rina Eiden for advice on statistics.

\section{References}

Adalbjarnardottir, S., \& Rafnsson, F. D. (2001). Perceived control in adolescent substance use: Concurrent and longitudinal analyses. Psychology of Addictive Behaviors, 15, 25-32.

Arbuckle, J. L., \& Wothke, W. (1999). AMOS 4.0 user's guide. Chicago: SPSS.

Beck, A. T., Steer, R. A., Ball, R., \& Ranieri, W. F. (1996). Comparison of Beck Depression Inventories-IA and -II in psychiatric outpatients. Journal of Personality Assessment, 67, 588-597.

Bentler, P. M. (1995). EQS: Structural equations programming manual. Encino, CA: Multivariate Software.

Bollen, K. A. (1989). Structural equations with latent variables. New York: Wiley.

Byrne, B. M. (2001). Structural equation modeling with AMOS: Basic concepts, applications, and programming. New Jersey: Erlbaum.

Chen, K., \& Kandel, D. (1995). The natural history of drug use from adolescence to mid-thirties in a general population sample. American Journal of Public Health, 85, 41-47.

Collins, R. L., Parks, G. A., \& Marlatt, G. A. (1985). Social determinants of alcohol consumption: The effects of social interaction and model status on the self-administration of alcohol. Journal of Consulting and Clinical Psychology, 53, 189-200.

Cooper, M. L., Frone, M. R., Russell, M., \& Mudar, P. (1995). Drinking to regulate positive and negative 
emotions: A motivational model of alcohol use. Journal of Personality and Social Psychology, 69, $990-1005$.

Costa, P. T., \& McCrae, R. (1991). NEO five-factor inventory (form S). Odessa, FL: Psychological Assessment Resources.

Costa, P. T., Terracciano, A., \& McCrae, R. (2001). Gender differences in personality traits across cultures: Robust and surprising findings. Journal of Personality and Social Psychology, 81, 322-331.

Dierker, L. C., Avenevoli, S., Stolar, M., \& Merikangas, K. R. (2002). Smoking and depression: An examination of mechanisms of comorbidity. The American Journal of Psychiatry, 159, 947-953.

Duncan, S. C., Duncan, T. E., \& Hops, H. (1998). Progressions of alcohol, cigarette, and marijuana use in adolescence. Journal of Behavioral Medicine, 21, 375-388.

Finn, P. R., Sharkansky, E. J., Brandt, K. M., \& Turcotte, N. (2000). The effects of familial risk, personality, and expectancies on alcohol use and abuse. Journal of Abnormal Psychology, 109, 122-133.

Friedman, H. S., Tucker, J. S., Schwartz, J. E., Martin, L. R., Tomlinson-Keasey, C., Wingard, D. L., \& Criqui, M. H. (1995). Childhood conscientiousness and longevity: Health behaviors and cause of death. Journal of Personality and Social Psychology, 68, 696-703.

Johnston, L. D., O’Malley, P. M., \& Bachman, J. G. (2002). Monitoring the future: National survey results on drug use, 1975-2001: Volume II. College students and adults ages 19-40. Washington, DC: U.S. Government Printing Office, NIH Publication No. 02-5107.

Katz, E. C., Fromme, K., \& D'Amico, E. J. (2000). Effects of outcome expectancies and personality on young adults' illicit drug use, heavy drinking, and risky sexual behavior. Cognitive Therapy and Research, 24, 1-22.

Locke, T., \& Newcomb, M. D. (2001). Alcohol involvement and dysphoria: A longitudinal examination of gender differences from late adolescence to adulthood. Psychology of Addictive Behaviors, 15, 227-236.

Nolen-Hoeksema, S., \& Girgus, J. S. (1994). The emergence of gender differences in depression during adolescence. Psychological Bulletin, 115, 424-443.

Patton, G. C., Hibbert, M., Rosier, M., Carlin, J. B., Caust, J., \& Bowes, G. (1996). Is smoking associated with depression and anxiety in teenagers. American Journal of Public Health, 86, 225-230.

Wagner, F. A., \& Anthony, J. C. (2002). Into the world of illegal drug use: Exposure opportunity and other mechanisms linking the use of alcohol, tobacco, marijuana, and cocaine. American Journal of Epidemiology, 155, 918-925.

Wills, T. A., Sandy, J. M., \& Yaeger, A. M. (2000). Temperament and adolescent substance use: An epigenetic approach to risk and protection. Journal of Personality, 68, 1127-1151.

Wills, T. A., Sandy, J. M., \& Yaeger, A. M. (2002). Stress and smoking in adolescence: A test of directional hypotheses. Health Psychology, 21, 122-130. 